\title{
Intratumoural expression of TNF-R1 and EMAP-II in relation to response of patients treated with TNF-based isolated limb perfusion
}

\author{
Remco van Horssen*, Joost A.P. Rens, Flavia Brunstein, Veronique Guns, Marjon van Gils, \\ Timo L.M. ten Hagen and Alexander M.M. Eggermont \\ Department of Surgical Oncology, Laboratory for Experimental Surgical Oncology, Erasmus MC - Daniel den Hoed \\ Cancer Centre, Rotterdam, The Netherlands
}

\begin{abstract}
Tumour necrosis factor-alpha (TNF) has been used in the clinic for more than 10 years in an isolated limb perfusion (ILP). However, intra-tumoural expression of TNF receptor-1 (TNF-R1) and TNF-R1 upregulating factors are unknown. We determined the expression of TNF-R1, proEMAP and endothelial monocyte-activating polypeptide-II (EMAP-II) before and after ILP and evaluated this against clinical response. Tumour biopsies were taken before and after ILP of patients $(n=27)$ with advanced sarcoma or metastatic melanoma. Biopsies were randomly analysed by western blotting for proEMAP/EMAP-II and TNF-R1 expression. Appropriate melanoma biopsies were stained for EMAP-II, TNFR1, CD31 and CD68. For melanomas we found that an up-regulation of EMAP-II, in contrast to proEMAP or TNF-R1, directly after ILP significantly correlated with a complete tumour response. No correlation was found for sarcoma patients. In a comparative analysis we found that the overall proEMAP and EMAP-II expression was higher in melanoma as compared to sarcoma cases and measurements in cell lines revealed high proEMAP expression by melanoma cells. We report high EMAP-II expression by endothelial cells and association with macrophages. In addition, macrophages are recruited to vessel-remnants after ILP. An upregulation of EMAP-II directly after ILP of melanoma patients correlates with and might predict a complete response to TNF-based ILP. The association of macrophages with EMAP-II expression and vascular damage suggests a role for EMAP-II in regulating the TNF-based anti-tumour effects observed with an ILP. Analysis of EMAP-II expression in melanoma biopsies should be implemented in the ILP procedure.

(C) 2006 Wiley-Liss, Inc.
\end{abstract}

Key words: TNF-R1; EMAP-II; ILP-patients

In the clinic, TNF is currently used in an isolated limb perfusion (ILP) setting and is registered in Europe since 1998. ${ }^{1}$ Patients with soft tissue sarcoma (STS) or in transit melanoma metastases in the extremities treated by an ILP with TNF and melphalan respond very well and $75 \%$ limb salvage is achieved with up to $80 \%$ response rates in patients with limb threatening locally advanced STS $^{2,3}$ whilst a $70 \%$ or higher complete response (CR) rate is achieved in patients with melanoma in transit metastases. ${ }^{4,5} \mathrm{Re}-$ sponding tumours show hemorrhagic necrosis and the TNF effect seems tumour associated vasculature-dependent. ${ }^{1}$ Molecular data on intra-tumoural expression of TNF-R1 and TNF-R1 upregulating factors however do not exist.

Endothelial monocyte-activating polypeptide-II (EMAP-II) is a multifunctional cytokine that was first isolated from supernatants of cultured murine fibrosarcoma and human melanoma cells. ${ }^{6,7}$ Even before it was named, its enhancing effects on tumour necrosis factor-alpha (TNF)-induced hemorrhagic tumour response were reported. ${ }^{8}$ EMAP-II induces chemo taxis of neutrophils and monocytes/macrophages and an induction of von Willebrand Factor, E- and P-selectin and Tissue Factor in endothelial cells. ${ }^{9,10}$ In addition, EMAP-II exhibits antiangiogenic activities by inducing endothelial cell apoptosis and suppression of tumour growth. ${ }^{11}$ Recently, a cancer cell dependent novel immunosuppressive role of EMAP-II by inducing lymphocyte apoptosis has been described. ${ }^{12}$ EMAP-II is transcribed in all cells as proEMAP and involved in protein translation as component of the tRNA synthase complex. ${ }^{13}$

For TNF-resistant tumours EMAP-II is shown to sensitise those tumours towards TNF upon EMAP-II gene transfer. These sensi- tising properties were not seen on tumour cells in vitro, suggesting an anti-tumour effect at vascular level. This was confirmed by a strong increase in Tissue Factor induction by high EMAP-II expressing tumours compared to wild type. ${ }^{14,15}$ Another relation between TNF and EMAP-II was found in endothelial cells in which EMAP-II was capable of inducing an up-regulation of TNF-R1 mRNA expression. ${ }^{16}$ However, this up-regulation on mRNA level did not correspond to protein levels and in a recent gene array study no records on EMAP-II-altered TNF-R1 expression were found ${ }^{17}$ suggesting that the relation between EMAP-II and TNF-R1 is more complex. In a rat model we showed that high EMAP-expressing tumours show a better response to TNF in an ILP. ${ }^{18}$ This TNF-sensitising effect of EMAP-II initiated studies on the role of EMAP-II in the clinical application of TNF in cancer treatment.

In the current study we show data on TNF-R1 and EMAP-II protein expression in tumours from patients treated with a TNF-melphalan ILP. In a double blind set-up we evaluated and quantified proEMAP, EMAP-II and TNF-R1 protein expression both before and after ILP of sarcoma and melanoma patients (total $n=27$ ). In addition, comparative analysis, histology and immunofluorescence stainings of melanoma to locate these proteins were done. The TNF-R1 and EMAP-II distribution within tumour tissue before and after ILP treatment indicates possible underlying mechanisms of the observed anti tumour effects of an ILP treatment.

\section{Material and methods \\ Patients}

At the Erasmus MC-Daniel den Hoed Cancer Centre more than 350 TNF-based ILPs were performed between 1991 and 2002 in soft tissue sarcoma and melanoma bearing patients. Because of the multifocality of the tumours and impossibility of surgical resection, all patients were potential candidates for amputation. Tumour material from these patients was stored in a tumour bank. Corresponding tumour biopsies before and after ILP were available for 11 patients. Only 2 melanoma patients received a melphalan alone ILP and no appropriate biopsies were available from these patients. Patients were included when a standardized taken biopsy was available both pre- and post-ILP. To expand this group, extra biopsies were taken from patients treated between 2001 and 2004. In total, 27 patients (14 suffering from sarcoma and 13 suffering from melanoma) were included in our study. Because of the nature of the biopsies, sarcomas were used for western blotting

Abbreviations: CR, complete response; EMAP-II, endothelial monocyte-activating polypeptide-II; ILP, isolated limb perfusion; LR, local recurrence; Met, metastases; PR, partial response; PT, primary tumour; SD, stable disease; STS, soft tissue sarcoma; TNF, tumour necrosis factoralpha; TNF-R1, TNF-receptor-1.

Grant sponsors: Erasmus MC-Translational Research Fund, Foundation

"Vanderes", Maxim Pharmaceuticals, Inc.

*Correspondence to: Erasmus MC, Laboratory for Experimental Surgical Oncology, Room Ee 0175 PO Box 1738, 3000 DR Rotterdam, The Netherlands. Fax: + 31-0-10-408-9471

E-mail: r.vanhorssen@erasmusmc.nl

Received 19 January 2006; Accepted after revision 10 February 2006

DOI $10.1002 /$ ijc. 21973

Published online 13 April 2006 in Wiley InterScience (www.interscience. wiley.com). 
TABLE I - PATIENT AND TUMOUR CHARACTERISTICS. THE 14 SARCOMA AND 13 MELANOMA PATIENTS CONSIST OF 15 FEMALES (56\%) AND 12 MALES (44\%), THE AVERAGE AGE IS 63 (RANGE 20-88)

\begin{tabular}{|c|c|c|c|c|c|c|c|c|c|c|}
\hline Pat. no. & Sex & Age (y) & Tumor type & Subtype sarcoma & Number of tumors & Site & Grade & Stage & ILP type & Response to ILP \\
\hline 1 & $\mathrm{~F}$ & 65 & $\mathrm{~S}$ & Pleiomorphic & $>10$ & Lower leg & High & PT & Fem & $\mathrm{SD}$ \\
\hline 2 & M & 74 & $\mathrm{~S}$ & Kaposi & $>10$ & Lower leg/foot & Low & PT & Fem & CR \\
\hline 3 & M & 74 & $\mathrm{~S}$ & Pleiomorphic & 1 & Upper arm & High & PT & Axil & $\mathrm{SD}$ \\
\hline 4 & M & 41 & $\mathrm{~S}$ & Lipo & 4 & $\begin{array}{c}\text { Upper/lower } \\
\text { leg, knee }\end{array}$ & High & LR & Iliac & PR \\
\hline 5 & M & 73 & $\mathrm{~S}$ & Leiomyo & $>10$ & Upper arm & High & LR & Axil & PR \\
\hline 6 & M & 54 & $\mathrm{~S}$ & Pleiomorphic & 1 & Upper leg & High & PT & Iliac & PR \\
\hline 7 & $\mathrm{~F}$ & 68 & $\mathrm{~S}$ & Leiomyo & 1 & Lower leg & High & PT & Fem & PR \\
\hline 8 & M & 88 & $\mathrm{~S}$ & Angio & 6 & Ankle/foot & Med & LR & Fem & CR \\
\hline 9 & M & 50 & $\mathrm{~S}$ & Neuro & 1 & Upper leg & Med & Met & Iliac & PR \\
\hline 10 & M & 20 & $\mathrm{~S}$ & Aggressive fibro & 8 & Upper/lower leg & Low & LR & Iliac & PR \\
\hline 11 & $\mathrm{~F}$ & 73 & $\mathrm{~S}$ & Clear & 1 & Upper leg & High & Met & Iliac & PR \\
\hline 12 & $\mathrm{~F}$ & 82 & $\mathrm{~S}$ & Mal fibr histiocyt & 1 & Upper leg & High & PT & Iliac & PR \\
\hline 13 & M & 51 & $\mathrm{~S}$ & Pleiomorphic & 1 & Upper arm & High & LR & Axil & PR \\
\hline 14 & $\mathrm{~F}$ & 45 & $\mathrm{~S}$ & Stuart-Treves & $>100$ & Upper leg & Med & PT & Iliac & PR \\
\hline 15 & $\mathrm{~F}$ & 82 & M & - & $>50$ & Lower leg & - & Met, PLN & Iliac & PR \\
\hline 16 & $\mathrm{~F}$ & 77 & M & - & $>100$ & Total leg & - & Met. PLN & Iliac & -1 \\
\hline 17 & M & 78 & M & - & $>100$ & Lower leg, foot & - & PT, Dmet & Iliac & PR \\
\hline 18 & $\mathrm{~F}$ & 62 & M & - & $<10$ & Lower arm & - & Met, PLN & Axil & $\mathrm{CR}$ \\
\hline 19 & M & 25 & M & - & $>100$ & Lower leg, foot & - & PT, Dmet & Iliac & PR \\
\hline 20 & $\mathrm{~F}$ & 57 & M & - & $>50$ & Total leg & - & Met, PLN & Iliac & CR \\
\hline 21 & M & 64 & M & - & $>20$ & Lower leg & - & Met, PLN & Fem & CR \\
\hline 22 & $\mathrm{~F}$ & 83 & M & - & $>50$ & Lower leg & - & Met, PLN & Fem & CR \\
\hline 23 & $\mathrm{~F}$ & 66 & M & - & $<10$ & Lower leg, foot & - & Met & Fem & CR \\
\hline 24 & $\mathrm{~F}$ & 65 & M & - & $>100$ & Total leg & - & Met, PLN & Iliac & SD \\
\hline 25 & $\mathrm{~F}$ & 77 & M & - & $<10$ & Lower leg, foot & - & Met, PLN & Iliac & PR \\
\hline 26 & $\mathrm{~F}$ & 76 & M & - & $>50$ & Total leg & - & Met & Iliac & CR \\
\hline 27 & $\mathrm{~F}$ & 46 & M & - & $>20$ & Total leg & - & Met, PLN & Iliac & $\mathrm{SD}$ \\
\hline
\end{tabular}

${ }^{1}$ Patient no. 16 died 4 days after ILP due to a myocardial infarction. This patient was included for expression studies but excluded from statistical analysis. Abbreviations: F, female; M, male; S, sarcoma; M, melanoma; Med, medium; PT, primary tumour; LR, local recurrence; Met, metastases; PLN, positive lymph nodes; Dmet, distal metastases; Fem, femoral ILP; Axil, axilar ILP; Iliac, Iliac ILP; SD, stable disease, CR, complete response; PR, partial response.

experiments and appropriate melanomas were used for immunofluorescence as well. TNF-based ILP with TNF and melphalan is a registered treatment modality for patients with limb threatening tumours, the protocols are approved by the Medical Ethical Committee of the Erasmus University Medical Centre and an informed consent form was obtained from all patients. Patients and tumour characteristics are listed in Table I.

\section{Isolated limb perfusion}

TNF-based ILP procedure has been described previously. ${ }^{2,3}$ Besides lymph node dissection, (for melanoma patients undergoing an iliac perfusion or with palpable lymph nodes) a tumour biopsy was taken directly before and directly after (within 12 minutes) the ILP procedure. The biopsies were directly frozen and stored at $-80^{\circ} \mathrm{C}$ until analysis. Response rates were reported according to WHO criteria ${ }^{19}$ in which a complete response (CR) is disappearance of all lesions with no new areas of disease appearing in the ILP regions. Partial response (PR) is defined as a reduction of $50-99 \%$ of the total tumour size and a stable disease (SD) is defined as no change after ILP treatment. The final outcome of the ILP consists of the clinical and histological response.

\section{Tissue lysis and Western blotting}

Tumour lysates were prepared in freshly made Lysis Buffer (50 mM Pipes/KOH, pH 6.5, 2 mM EDTA, 0.1\% Chaps, $5 \mathrm{mM}$ DTT, protease inhibitor cocktail (Roche Diagnostics)), $100 \mu \mathrm{l}$ per $25 \mathrm{mg}$ tumour-tissue and the samples were subjected to 3 freezethaw cycles. Cultured tumour cells were lysed as described later. Samples were centrifuged for $10 \mathrm{~min}$ at $14,000 \mathrm{rpm}$ at $4^{\circ} \mathrm{C}$ and within the clear supernatant the protein concentration was determined using Coomassie Reagent (Uptima). One hundred micrograms of tumour lysate or $150 \mu \mathrm{g}$ of total protein from cultured cells was resolved by SDS-PAGE, transferred to polyvinylidene difluoride membranes (BioRad) and analysed by Western blotting using rabbit anti-human EMAP-II (BioSource) or mouse antihuman TNF-R1 (R\&D Systems). Expression was quantified by densitometric analysis using ImageJ Software (NIH) and compared to positive control (recombinant protein) for EMAP-II (BioSource) or expressed as absolute total density (averaged pixel value times the number of pixels) for TNF-R1.

\section{Cell culture}

B16BL6 (mouse melanoma), BLM (human melanoma) and Mel57 (human melanoma) cells were cultured in DMEM medium (Biowhittaker), BN175 (rat syngeneic soft tissue sarcoma) cells were cultured in RPMI 1640 medium (Biowhittaker), ROS-1 (rat osteo sarcoma) cells were cultured in EMEM medium supplemented with $10 \%$ Fetal Calf Serum (FCS) and $100 \mathrm{U} / \mathrm{ml}$ penicillin/streptomycin (Life Technologies). Tumour cells were maintained at $37^{\circ} \mathrm{C}, 5 \% \mathrm{CO}_{2}$ in a humidified incubator and routinely subcultured by removal from flasks using Trypsin-EDTA (SigmaAldrich). Human umbilical vein endothelial cells (HUVEC) were used between passage 3 and 7 and maintained in Human endothelial-SFM medium supplemented with $10 \%$ new-born calf serum, $5 \%$ Human Serum (Invitrogen), $20 \mathrm{ng} / \mathrm{ml} \mathrm{bFGF} \mathrm{and} 100 \mathrm{ng} / \mathrm{ml}$ EGF (PeproTech), in gelatin-coated flasks.

\section{ELISA}

Total EMAP concentrations were determined in tumour cell lysates. Tumour cells were counted and lysed in $75 \mu \mathrm{l} / 1.10^{6}$ cells Lysis Buffer (50 mM Tris- $\mathrm{HCl}(\mathrm{pH} \mathrm{7,4),} 150 \mathrm{mM} \mathrm{NaCl}, 1 \mathrm{mM}$ EDTA, $1 \%$ NP-40, protein inhibitor cocktail) for $30 \mathrm{~min}$ on ice. Cell lysates were cleared from cellular debris by centrifugation for $10 \mathrm{~min}$ at $0^{\circ} \mathrm{C}, 14,000 \mathrm{rpm}$ and stored at $-80^{\circ} \mathrm{C}$ until analysis. ELISA was performed using the Accucyte Human EMAP-2 Kit for detection of total EMAP-2 (CytImmune) using the provided reagents according to the manufacturers instructions.

\section{Immunofluorescence stainings}

Cryosections $(5 \mu \mathrm{m})$ of Tissue-Tek (Sakura) embedded melanoma biopsies were fixed on polylysine-coated slides with acetone, air-dried and rehydrated with PBS. Tissue sections were 


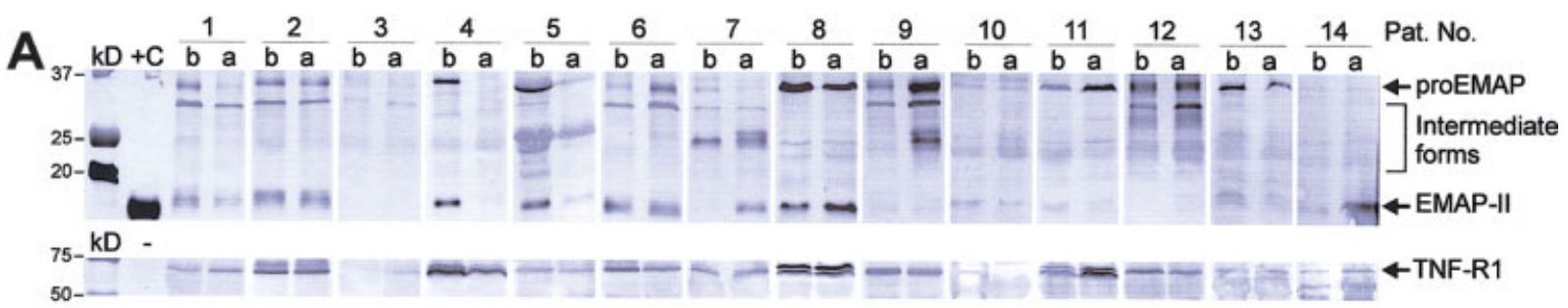

B

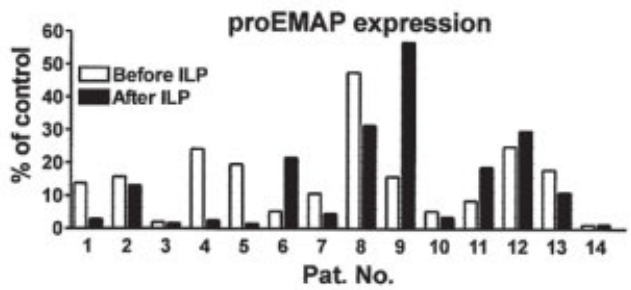

D

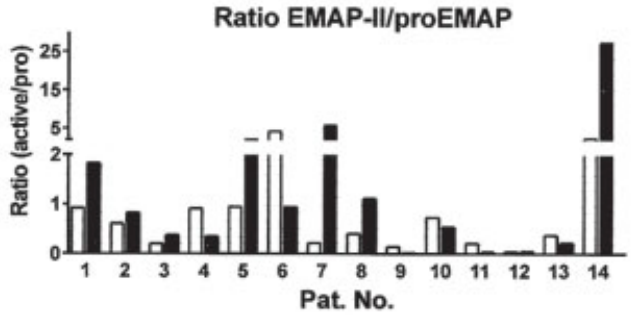

C

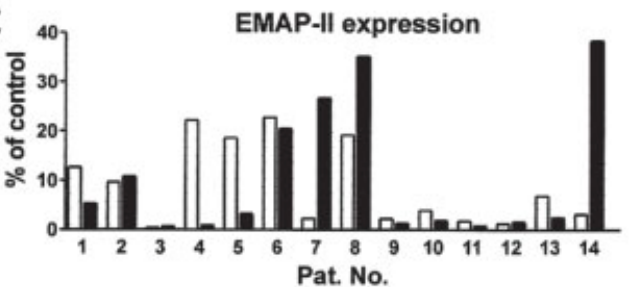

$\mathbf{E}$

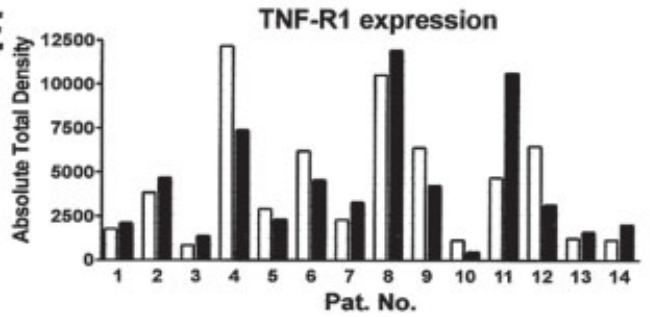

Figure 1 - Western blot analysis of EMAP-II and TNF-R1 expression in tumour biopsies from sarcoma patients taken right before (b) and after (a) TNF-based ILP. (a) Western blots. + C, 50 ng recombinant EMAP-II protein, for TNF-R1 no positive control was available. (b) Quantification of proEMAP expression before (open bars) and after (closed bars) ILP as compared to the positive control. (c) Quantification of EMAP-II expression before and after ILP as compared to the positive control. (d) Ratio of EMAP-II/proEMAP derived from $(b)$ and $(c)$ before and after ILP. (e) Quantification of TNF-R1 expression before and after ILP shown as absolute density values.

incubated in blocking solution (1\% BSA/0.05\% Tween-20/PBS) for at least $30 \mathrm{~min}$ and subsequently incubated with primary antibodies (rabbit anti-human TNF-R1, R\&D Systems, mouse antihuman TNF-R1, Alexis, rabbit anti-human EMAP-II, BioSource, sheep anti-human CD31, R\&D Systems, mouse anti-human CD68, DaKo Cytomation) for $1 \mathrm{hr}$ at room temperature (CD31, CD68) or overnight at $4^{\circ} \mathrm{C}$ (EMAP-II, TNF-R1) followed by green (488) and red (594) labelled Alexa Fluor secondary antibodies (Molecular Probes) for $1 \mathrm{hr}$ at room temperature. Specificity of the antibodies used was checked by staining with secondary antibodies alone. Slides were mounted using a 1:1 solution of VectaShield (Vector Laboratories) and DAPI-DABCO (Molecular Probes). Immunofluorescent images were taken using an Axiovert $100 \mathrm{M}$ microscope with $40 \times / 1.30$ Oil-Plan-NEOFLUAR objective lens (Carl Zeiss) and an ORCA II ER camera (C4742-98, Hamamatsu Photonics Systems) with Openlab 3.1.5 software (Improvision).

\section{HE staining}

The same tumour biopsies of melanoma patients used for western blot analysis were used for histological analysis. Five-micrometre cryosections of Tissue-Tek (Sakura) embedded melanoma biopsies were fixed on polylysine-coated slides with acetone, airdried and rehydrated with PBS. Sections were stained with haematoxylin and eosin using standard procedures. Slides were examined on a Leica DM-RXA microscope and photographed using a Sony 3CCD DXC 950 camera.

\section{Statistical analysis}

ProEMAP, EMAP-II and TNF-R1 expression were divided in up- and down regulated after ILP treatment. Expression threshold was set at $>5 \%$ compared to control and an upregulation was considered relevant when it was at least 1.2 times higher compared to before treatment. ProEMAP and EMAP-II were compared to TNF-R1 expression and clinical outcome using cross tabulation and Chi-Square tests. All tests were 2 sided and the cutoff level for statistical significance was set at $p<0.05$.

\section{Results}

Patients

Twenty-seven patients were included in our study, divided in 14 suffering from sarcoma and 13 suffering from melanoma confined to leg or arm. All patients were treated with an ILP with a combination of TNF and melphalan. Patients were included when a standardized taken tumour biopsy was available from just before and immediately after ILP. From 1 patient (no. 21) also normal skin biopsies were taken. From 1 patient (no. 16) no response data were available because this patient died 4 days after a leakage-free ILP due to a myocardial infarction and was known with a previous cardiac history. Patient characteristics are summarized in Table I.

\section{EMAP-II and TNF-RI expression before and after ILP in sarcoma patients}

Figure 1a shows protein expression of EMAP-II and TNF-R1 in 14 sarcoma patients before (b) and after (a) ILP. All sarcoma patients show proEMAP expression although in patient 3 and 14 the expression is very low. The mature cytokine EMAP-II was also observed in nearly all sarcoma biopsies although it was very low in a number of patients. In addition some intermediate forms were observed which is in accordance with previous reports. ${ }^{20,21}$ Also TNF-R1 expression was found in almost all sarcoma patients. For proEMAP, EMAP-II and TNF-R1 protein expression varied a lot between patients and before and after ILP. ProEMAP and EMAP-II expression were quantified using densitometric analysis and illustrated as percentage of control (recombinant 


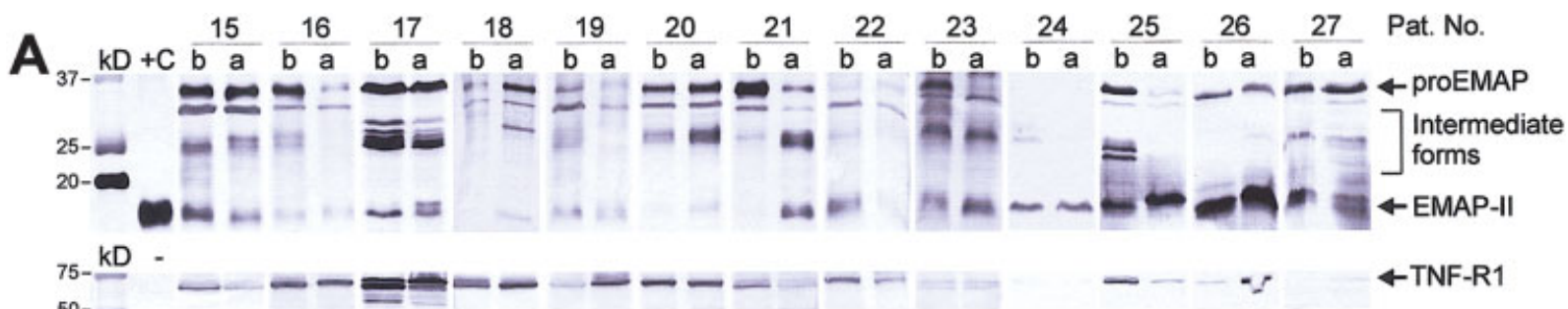

B
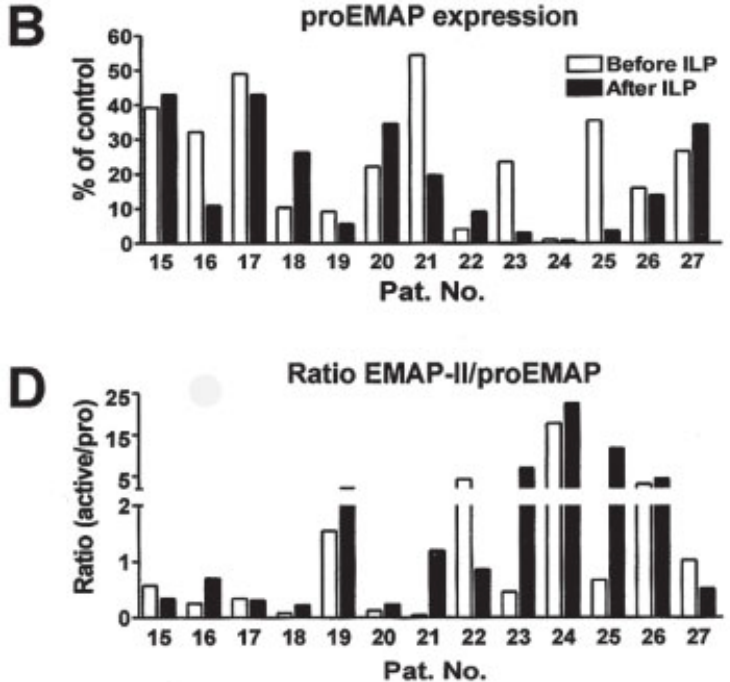
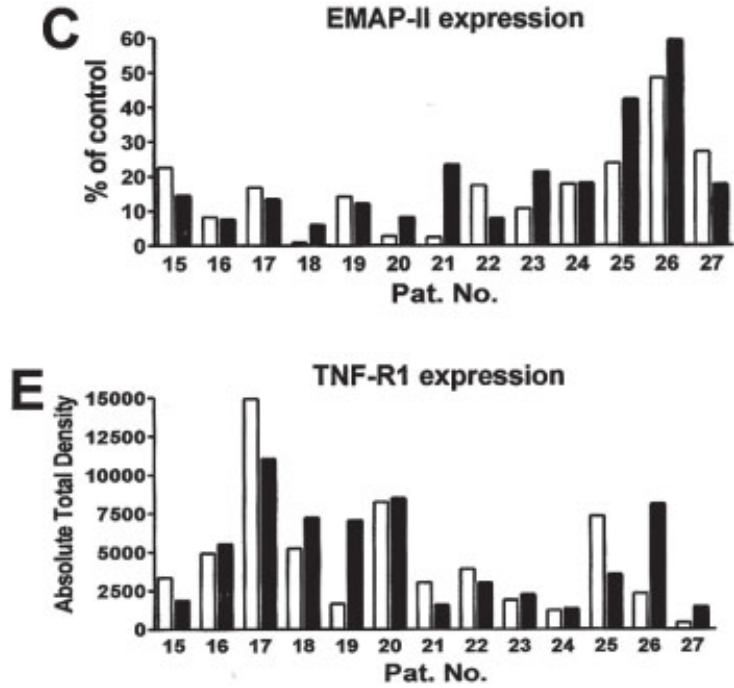

FigURE 2 - Western blot analysis of EMAP-II and TNF-R1 expression in tumour biopsies from melanoma patients taken right before (b) and after (a) TNF-based ILP. (a) Western blots. + C, 50 ng recombinant EMAP-II protein, for TNF-R1 no positive control was available. (b) Quantification of proEMAP expression before (open bars) and after (closed bars) ILP as compared to the positive control. (c) Quantification of EMAPII expression before and after ILP as compared to the positive control. $(d)$ Ratio of EMAP-II/proEMAP derived from $(b)$ and $(c)$ before and after ILP. (e) Quantification of TNF-R1 expression before and after ILP shown as absolute density values.

EMAP-II) before and after ILP (Figs. $1 b$ and $1 c$ ). Because activities are attributed to the active form, as a derivative of these quantitative data the ratio EMAP-II/proEMAP was calculated (Fig. 1d). The quantification of TNF-R1 expression is illustrated as total absolute density because no positive control was available (Fig. 1e).

\section{EMAP-II and TNF-RI expression before and after ILP in melanoma patients}

For 13 melanoma patients (patient 15-27) the same analyses were performed as for the sarcoma patients. Except for patient 24, all melanoma patients show proEMAP expression and EMAP-II was found in all biopsies (Fig. 2a). Similar to the sarcoma patients some intermediate forms were observed as well. TNF-R1 expression was found in all melanoma patients although very low in patient 24 and 27. Quantitative analysis of proEMAP and EMAPII expression was done and expressed as percentage of control (Figs. $2 b$ and $2 c$ ). The derived ratio EMAP-II/proEMAP is shown in Figure $2 d$ and the absolute total density values for TNF-R1 expression in the melanoma patients are depicted in Figure $2 e$. When comparing Figures $1 a$ and $2 a$, the expression of proEMAP and EMAP-II in melanoma biopsies was more abundant than in sarcoma biopsies.

\section{Correlation analysis}

To demonstrate statistical correlation between proEMAP, EMAP-II and TNF-R1 expression and response rates we performed Chi-Square analysis. We divided the patients in up- and down-regulation of proEMAP, EMAP-II and TNF-R1 protein expression upon ILP treatment. An upregulation of 1.2-fold and expression of $>5 \%$ were set as thresholds. A 1.2 increase was considered relevant in terms of measurements used and to prevent
TABLE II - CORRELATION STATISTICS SHOWING p-VALUES OF CHI-SQUARE ANALYSIS. ALL TESTS WERE TWO SIDED AND THE CUT-OFF LEVEL FOR STATISTICAL SIGNIFICANCE WAS SET AT $\mathrm{p}<0.05$. ARROW UP MEANS $>1.2$ TIMES UP-REGULATION AFTER ILP TREATMENT

\begin{tabular}{lcc}
\hline & \multicolumn{2}{c}{ MELANOMA } \\
\cline { 2 - 3 } & TNF-R1 $\uparrow$ & $\mathrm{CR}$ \\
\hline proEMAP $\uparrow$ & 0.433 & 0.260 \\
EMAP-II $\uparrow$ & 1.000 & 0.018 \\
Ratio (act/pro) & 0.699 & 0.260 \\
TNF-R1 $\uparrow$ & - & 1.000 \\
\hline
\end{tabular}

inclusion of observed upregulations that are too small to be biologically relevant. Whether this 1.2 increase is biologically relevant in itself cannot be concluded but local upregulation is known to be of crucial importance for EMAP-II to exert its biological activities (see discussion section). In addition, the patients were divided in complete responders (CR) at 1 site and partial responders (PR) or stable disease (SD) at the other. The successes (up-regulation and $\mathrm{CR}$ ) were correlated to the failures (down regulation and PR/SD) for sarcoma and melanoma patients by cross tabulation and Chi-Square analysis. The $p$-values for significance are shown in Table II. An up-regulation of proEMAP or the ratio did not correlate with an up-regulation of TNF-R1 or a CR for melanoma patients. In contrast, a significant correlation was found between an up-regulation of EMAP-II and a CR for melanomas $(p=0.018)$. Thus, when EMAP-II is upregulated directly after ILP treatment, the patient is expected to have a CR. Up-regulation of EMAP-II therefore could be an independent prognostic marker for a CR of melanoma patients. Between an up-regulation of TNF$\mathrm{R} 1$ and CR no relation was found (Table II). For sarcoma patients no positive correlation was found. 


\begin{tabular}{|c|c|c|c|c|c|c|}
\hline \multirow{2}{*}{$\mathbf{A}$} & \multicolumn{3}{|c|}{ BEFORE ILP } & \multicolumn{3}{|c|}{ AFTER ILP } \\
\hline & Sarcon & & Melanoma & Sarco & & Melanc \\
\hline ProEMAP (\%) & 15.2 & & 24.9 & 14.3 & & 19.0 \\
\hline EMAP-II (\%) & 9.1 & & 16.4 & 10.7 & & 19.3 \\
\hline \multirow[t]{2}{*}{$\mathbf{B}$} & \multicolumn{6}{|c|}{ SARCOMA } \\
\hline & \multicolumn{3}{|c|}{ Before ILP } & \multicolumn{3}{|c|}{ After ILP } \\
\hline Grade & Low & Med & High & Low & Med & High \\
\hline ProEMAP (\%) & 10.6 & 21.6 & 14.1 & 8.3 & 29.9 & 10.5 \\
\hline EMAP-II (\%) & 6.8 & 8.2 & 9.9 & 6.4 & 25.0 & 6.9 \\
\hline Stage & PT & LR & Met & PT & LR & Met \\
\hline ProEMA & 10.6 & 23.0 & 12.2 & 10.7 & 10.0 & 37.8 \\
\hline EMAP-II (\%) & 7.5 & 14.2 & 2.0 & 14.9 & 8.7 & 1.1 \\
\hline
\end{tabular}
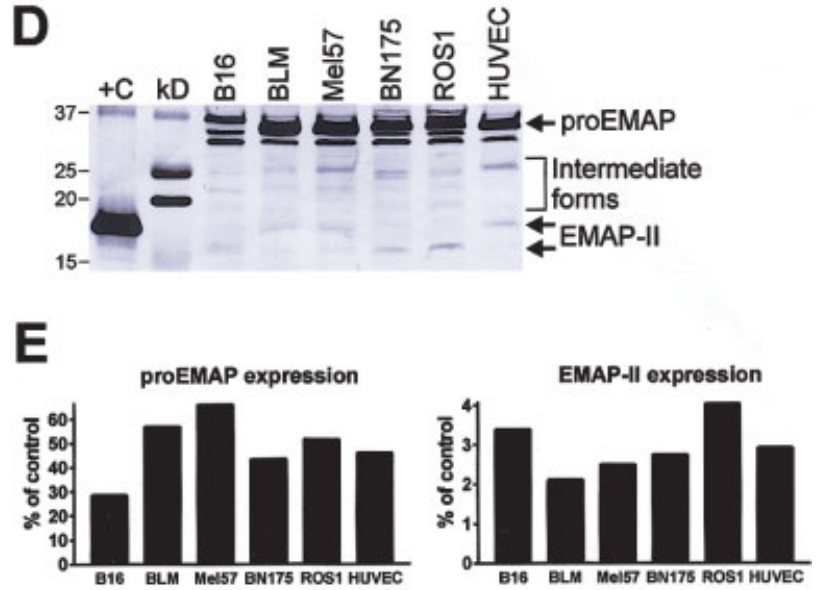

\begin{tabular}{lllllll} 
C & \multicolumn{4}{c}{ MELANOMA } \\
\cline { 2 - 7 } & \multicolumn{3}{c}{ Before ILP } & \multicolumn{3}{c}{ After ILP } \\
\cline { 2 - 7 } Stage & Met,PLN PT,Dmet & Met & Met,PLN PT,Dmet & Met \\
\hline ProEMAP (\%) & 25.0 & 29.2 & 19.7 & 20.2 & 24.3 & 8.5 \\
EMAP-II (\%) & 13.6 & 15.5 & 29.6 & 16.1 & 12.8 & 40.4
\end{tabular}

\begin{tabular}{ll} 
& \\
Celline & EMAP $(\mathrm{ng} / \mathrm{ml})$ \\
\hline B16 & 420 \\
BLM & 580 \\
Mel57 & 660 \\
BN175 & 320 \\
ROS1 & 26 \\
HUVEC & 460
\end{tabular}

Figure 3 - Comparative analysis of proEMAP/EMAP-II expression in melanoma and sarcoma tumours. (a) Table showing overall relative proEMAP/EMAP-II expression in the sarcoma and melanoma biopsies before and after ILP. In melanoma all relative values are higher as compared to sarcoma. (b) ProEMAP/EMAP-II expression in sarcoma patients subdivided by grade or stage of the tumour. For abbreviations see legend Table I. $(c)$ ProEMAP/EMAP-II expression in melanoma patients subdivided by stage of the tumour. Note the high EMAP-II expression in 2 patients with metastases after ILP. For abbreviations see legend Table I. $(d)$ Western blot analysis of EMAP expression in tumour cell lines. Three melanomas (B16, BLM, Mel57) were compared with 2 sarcoma lines (BN175, ROS1). HUVEC were used as EC control. (e) Quantification of western blot analysis showing the relative proEMAP/EMAP expression as compared to positive control. Note the very low EMAP-II expression of the cultured (unstimulated) cells. $(f)$ Total EMAP concentration in tumour cell lysates measured by ELISA.

Comparative analysis of proEMAP/EMAP-II expression in sarcoma and melanoma

An upregulation of EMAP-II did correlate with CR in melanoma patients but not in sarcoma patients. Comparing these 2 groups for relative proEMAP and EMAP-II expression revealed that for both proEMAP and EMAP-II, both before and after ILP, melanoma tumour biopsies had a higher relative expression (Fig. $3 a$ ). The differences were most profound for EMAP-II (in melanoma 1.8 times higher before and after ILP), proEMAP was 1.6 and 1.3 times higher in melanoma before and after ILP respectively. For a more detailed analysis we also compared subgroups within sarcoma and melanoma patients. Sarcoma cases were divided according to grade and stage (Fig. $3 b$ ). Although some subgroups were very small, some interesting differences were observed. Both before and after, ILP proEMAP was the highest in medium-grade sarcomas while for EMAP-II this only was the case after ILP. The abundant upregulation of EMAP-II after ILP in the medium-grade sarcomas (from 8.2 to $25 \%$ ) resulted from 3 patients (no. 8, 9 and 14) with $1 \mathrm{CR}$ and $2 \mathrm{PR}$. The 2 SD patients (no. 1 and 3) were both high-grade sarcomas and for this group a downregulation for EMAP-II after ILP was found. When comparing the different stages before ILP proEMAP was the highest in the local recurrence (LR) patients, while after ILP patients with metastases (Met) had highest proEMAP expression (Fig. 3b, lower panel). However, EMAP-II expression in the Met-subgroup was almost absent while the 2 patients (no. 9 and 11) did have a PR. For Primary Tumours (PT) we observe the most abundant upregulation of EMAP-II after ILP (from 7.5 to 14.9\%) but although most of the PT sarcoma patients had a PR also 2 patients of this group had a SD.
Melanoma patients were divided according to stage (Fig. 3c). For all subgroups, except for the Met group after ILP, the proEMAP expression was very high. Interestingly, for EMAP-II the opposite was the case: EMAP-II expression was very high after ILP in the Met patient group (40.4\%). Patients (no. 23 and 26) within this group did have a CR although also before ILP the EMAP-II expression was already high in these 2 patients. In conclusion, we observed higher proEMAP/EMAP-II levels in melanoma patients and although these groups were too small to draw any conclusions, some interesting differences were found between the subgroups.

\section{ProEMAP/EMAP-II expression in tumour cell lines}

For a further analysis of cellular proEMAP/EMAP-II expression by melanoma and sarcoma cells we evaluated the expression in some melanoma and sarcoma cell lines available in the lab. Using quantitative western blotting the 2 human melanoma cell lines analysed (BLM, Mel57) had the most abundant proEMAP expression although the differences with ROS-1 and HUVEC were small (Figs. $3 d$ and $3 e$ ). The relatively high proEMAP levels in the endothelial cell line did correspond with the co-staining experiments (see later). Surprisingly, we observed differences between species of EMAP-II active forms. For the human cells (BLM, Mel57 and HUVEC) the apparent molecular weight was $18-19 \mathrm{kD}$ while for the mouse and rat lines (B16, BN175 and ROS-1) it was around $16-17 \mathrm{kD}$. For all cell lines the EMAP-II expression was very low (Fig. $3 e$, right graph). In addition, ELISA was performed for total EMAP within lysates of tumour cells. For Mel57 and BLM melanoma lines EMAP concentration was higher as compared to the 
other cell lines. The values for the sarcoma lines BN-175 and ROS-1 were lower and very low respectively as determined by the ELISA procedure (Fig. $3 f$ ).

\section{Immunofluorescent detection of EMAP-II and TNF-RI in melanoma biopsies}

Double fluorescent staining for EMAP-II and TNF-R1 expression before and after ILP treatment are shown in Figure $4 a$. Before ILP it is obvious that all cells express proEMAP-EMAP-II due to the function of proEMAP in protein translation. In some vessellike regions throughout the biopsy a higher expression was found (arrow). Before ILP, TNF-R1 expression was found in a fraction of the cells while these high TNF-R1 expressing cells located nearby the vessel-like structures. Evidently, the high expressing EMAP-II cells and the high expressing TNF-R1 cells do not overlap as shown in the merged image (Fig. $4 a$, upper panel). After ILP the tumour structure is completely disrupted and no vessellike structures were observed anymore. EMAP-II expression was still seen in all cells and some clustering of high EMAP-II expressing cells was noted (Fig. $4 a$, lower panel). TNF-R1 expression after ILP was found in a fraction of the cells and its expression seemed reorganized as receptor-clusters (arrowheads). The high expressing EMAP-II cells after ILP did not correlate with the high expressing TNF-R1 cells. To compare these immunofluorescence data with tumour histology HE stainings were done of the same biopsies (Fig. 4b). Before-ILP (upper panel) heterogeneous tumour histology is observed with characteristics of malignant melanoma like differences in tumour nuclei dimensions, tumour infiltrate and tumour-associated vessels (asterisk). The right panel shows an example of melanoma vessel(s) before ILP. After ILP (lower panel) the tumour histology is reorganised, cells that have a macrophage appearance tend to cluster (asterisk). The vessel in the right panel shows also association with infiltrate-like cells.

\section{Localization of EMAP-II and TNF-RI in melanoma biopsies before and after ILP}

To localize EMAP-II and TNF-R1 expression in the heterogeneous tumour sections we co-stained EMAP-II and TNF-R1 together with endothelial (CD31) and macrophages/monocytes marker (CD68) before and after ILP (Fig. 5). As shown in Figure $5 a$, the vessel-like structures found with the EMAP-II staining in Figure 4 indeed (in part) co-stained with CD31. These endothelial cells had a high EMAP-II expression before ILP (Fig. 5a, arrow). In contrast, TNF-R1 expressing cells did not co-stain with the endothelium (Fig. 5a, upper panel). Before ILP, cells with high TNF-R1 expression located in close proximity to vessels, confirming our results shown in Figure 4. After ILP, within the disrupted tumour structure high expressing EMAP-II cells were still present clustered together. The CD31 positive cells stained positive for EMAP-II as well. TNF-R1 expression after ILP was again found in receptor-clusters (Fig. $5 a$, arrowheads) and the endothelial cells expressed TNF-R1 at very low levels. Co-staining with CD68 revealed that high EMAP-II expressing cells or regions were associated with macrophages. Moreover, this co-staining was in close proximity to vessel-like structures (Fig. $5 b$, arrow). The high TNF-R1 expressing cells in the melanoma tissue before ILP were macrophages; a strong co-staining was observed for TNF-R1 and CD68 (Fig. $5 b$, asterisks). After ILP, the association of EMAP-II expression and macrophages was still abundant, with similar staining-patterns. For TNF-R1, association with CD68 positive cells was less after ILP as compared to before treatment (Fig. 5b, lower panel, arrowheads point receptor-clusters). Finally, we stained melanoma tumour biopsies for CD31 and CD68 together (Fig. 5c). Before ILP, healthy-looking vessels are present and macrophages were distributed quite equally throughout the tumour tissue. After ILP vessels appeared disrupted and macrophages were present in close proximity to these vessel remnants.

\section{EMAP-II and TNF-RI expression in normal skin and melanoma}

From 1 melanoma patient (no. 21) besides a melanoma biopsy also a biopsy of normal skin was taken before and after TNFbased ILP. EMAP-II and TNF-R1 expression were determined by quantitative western blotting. Strikingly, in normal skin, exactly the opposite occurred compared to the melanoma (Fig. 6a). In the normal skin the ILP treatment induced an up-regulation of proEMAP and a down-regulation of EMAP-II, while in the melanoma EMAP-II is upregulated while proEMAP is lower after ILP. Quantification is shown in Figures $6 b$ and $6 c$. The ratio shows the same opposite effects for the normal skin versus the melanoma tumour (Fig. 6d). For TNF-R1 also an opposite effect was found; TNF-R1 expression was high after ILP in the normal skin and before ILP in the melanoma tumour (Fig. 6a).

\section{Discussion}

Here we describe novel data on TNF-R1 and EMAP-II protein expression in cancer patients treated with a TNF-based ILP. For melanoma we report an association between EMAP-II and the response to the TNF-ILP treatment. Our data strongly complement previous knowledge from preclinical and fundamental studies on EMAP-II and might have important implications for patients treated with a TNF-based ILP.

Out of the 350 TNF-based ILPs performed at our institute we selected patients based on the availability of tumour tissue. For our study we needed tumour tissue taken directly before and directly after ILP. Therefore this patient group is unique. The response rates of the sarcoma and melanoma patients in our patient group nicely matches the response rates of larger groups treated by TNF-based ILP. ${ }^{3,22}$ For sarcomas our study group had $2 \mathrm{CR}(14 \%)$, which is somewhat lower as compared to larger cohorts and most likely caused by the relative small group $(n=$ 14) used in this mechanistic study. The overall response rate is $86 \%$ and comparable with other studies.

We employed both immunologic staining and Western blot analysis, to firstly analyse TNF-R1, proEMAP and EMAP-II expression levels before and after treatment, in a quantitative manner (Figs. 1 and 2), and secondly to provide insight in localization of the proteins within the tumour tissues.

Because of the nature of the biopsies, no histological analysis was possible on sarcoma material and only a fraction of the melanoma biopsies can be used. These 3-5 patients were not representing the whole cohort so findings need to be justified as supportive and not conclusive observations. Using these melanoma biopsies we found proEMAP/EMAP-II staining in all cells. In some regions of the tumour we observed higher expression levels, which might be due to an up regulation of protein synthesis but, based on costaining, we conclude that these regions contained a higher level of the secreted active EMAP-II cytokine. The high expression patterns have a vessel-like structure (Fig. 4), which we could confirm when co-stained with CD31 (Fig. 5a) and is in agreement with other reports. ${ }^{23}$ For tumour histology analysis we also were restricted to the same melanoma material because we preferred to have biopsies both directly pre- and post-ILP and only frozen material was available, resulting in sections with some gaps. Histological experiments confirmed the reorganisation of tumour cells. In HE sections after ILP we observed infiltrate-like cells close to the vessels and based on specific staining we concluded that these infiltrate contained macrophages.

Surprisingly, to our knowledge no literature is available on TNF-R1 distribution within tumour tissue before and after ILP treatment. Previously, measurements on TNF-R1 were done in serum of patients to determine the concentration of soluble TNF-R1 in response to TNF-based ILP. ${ }^{24,25}$ Here we show for the first time TNF-R1 protein distribution in tumour tissue before and after ILP. We found that TNF-R1 is mainly expressed by cells located closely to vessels but not by endothelial cells themselves. After ILP the vascular structure is disrupted and for TNF-R1 we 

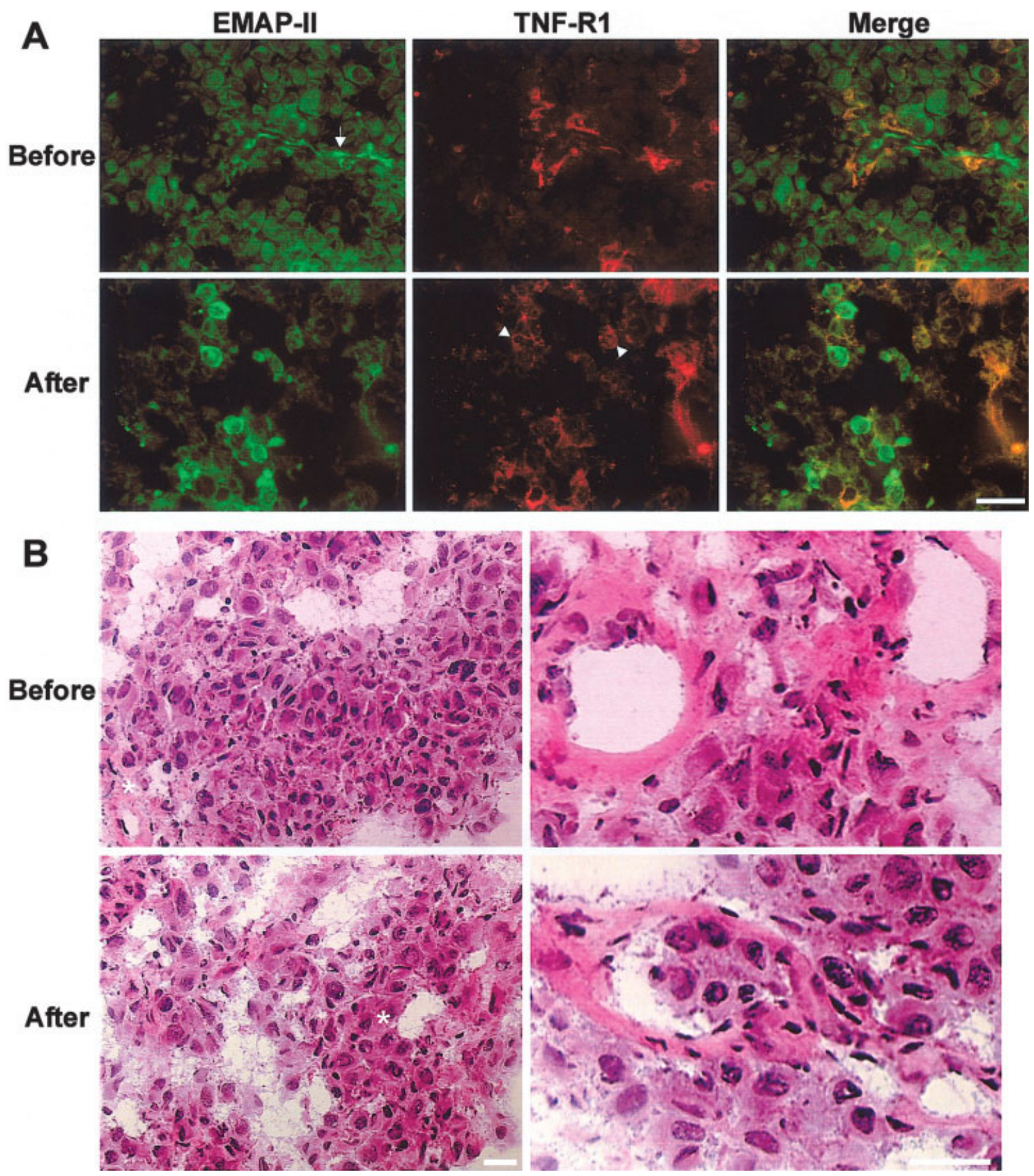

FIgURE 4 - (a) Immunofluorescence stainings for EMAP-II (green) and TNF-R1 (red) of melanoma biopsies taken right before (upper panel) and after (lower panel) TNF-based ILP. Before ILP proEMAP-EMAP-II is present in all cells but some vessel-like regions (arrow) have a higher expression, likely due to local increased expression of mature EMAP-II. TNF-R1 is mainly expressed in the surrounding of these vessel-like regions in a scattered manner. Noteworthy is the observation that high EMAP-II expressing cells and TNF-R1 expressing cells de not overlap. After ILP the tumour structure is extensively damaged. Some high EMAP-II expressing tumour cells clustered to together were observed. TNFR1 expression was still present in some tumour cells and appeared more organized in clusters (arrowheads) as compared to before ILP. The vessel-like structures are not visible anymore after ILP. Bar, $50 \mu \mathrm{m}$. (b) Histological HE staining of melanoma biopsies taken before and after ILP. Before ILP the melanoma showed a heterogeneous structure of tumour cells and infiltrate (left panel). After ILP the tumour cells are strongly redistributed and the tumour-associated macrophages (also called foamy macrophages) tend to cluster, asterisk. Tumour vessels before and after ILP are shown at the right panel. Bar, $20 \mu \mathrm{m}$.

observed a cluster-like pattern, a feature reported for other members of the death receptor family as well. ${ }^{26}$ Expression level of TNF-R1 was however not changed by the perfusion, suggesting that rather protein redistribution than up- or down-regulation of TNF-R1 is involved in the anti-tumour effects of TNF.
To attribute expression of EMAP-II and TNF-R1 protein to specific cells within the heterogeneous tumour tissue we performed co-staining experiments with endothelial and macrophage/monocyte markers (Fig. 5). Endothelial cells were chosen because of the known anti-vascular activities of TNF in an ILP and the 

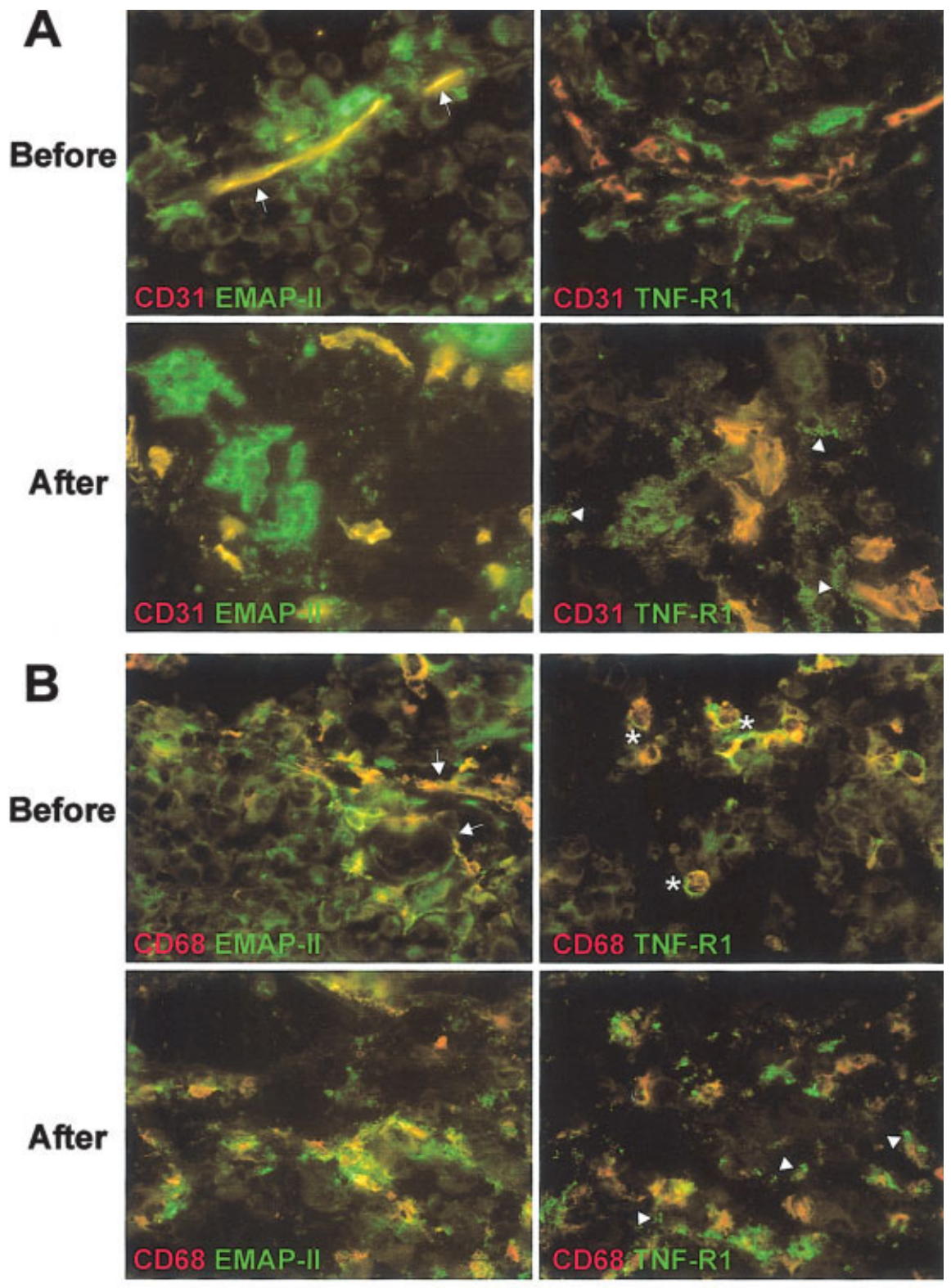

Figure 5-Immunofluorescence stainings for EMAP-II and TNF-R1 in combination with endothelial marker (CD31, panel a) or macrophage/monocyte marker (CD68, panel $b$ ) and co-staining with both markers (CD31/CD68, panel c) of melanoma biopsies taken right before and after TNF-based ILP. (a) Before ILP the high EMAP-II expressing regions in part co-localize with endothelial cells (arrow) while the TNF-R1 expressing cells are surrounding the vessels. No co-localization of TNF-R1 with endothelial cells was observed. After ILP high expressing EMAP-II cells were still present and endothelial cells of the disrupted vessels stained positive as well. TNF-R1 was observed in clusters (arrowheads) and some expression was seen in endothelial cells. (b)

Before

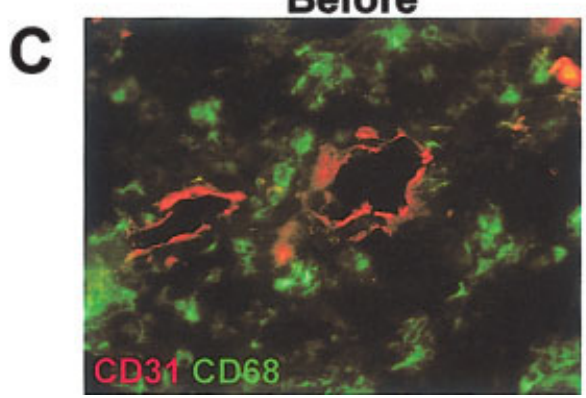

After

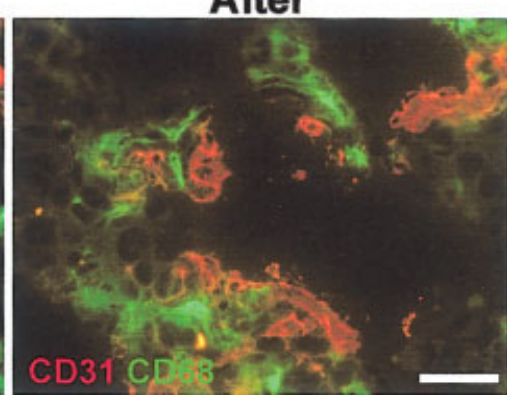

Before ILP, high expressing EMAP-II cells/ regions co-stained with macrophages in vessel-like regions (arrow). Most tumour-macrophages expressed high levels of TNF-R1 (asterisks). After ILP co-localization of EMAP-II and macrophages is obvious while clustered TNF-R1 expression (arrowheads) was less associated with macrophages. $(c)$ Before ILP, healthy-looking vessels are present and macrophages are equally distributed throughout the tumour. After ILP the tumour vasculature has been disrupted and macrophages were recruited to these endothelial/vessel remnants. reported anti-angiogenic properties of EMAP-II ${ }^{1,27}$ and macrophages/monocytes are very well known for their contribution in tumourgenesis and are one of the major responsive cells to the chemo attractive activities of EMAP-II. ${ }^{28,29}$ Co-staining of endothelial cells with EMAP-II implies that high EMAP-II expression may be involved in the anti-vascular effects of TNF. The absence of TNF-R1 on tumour endothelium was unexpected. Only after
ILP little positive staining was observed. For endothelial cells in vitro it is known that they express TNF-R1 and for EMAP-II an upregulating role has been suggested. ${ }^{16}$ It is known that TNF-R1 expression is mainly in the Golgi apparatus and only very small (signalling) fractions are present on the membrane. ${ }^{30}$ However, also Golgi-staining was not observed. This might be caused by the tissue staining procedures, but this also may reflect a major differ- 


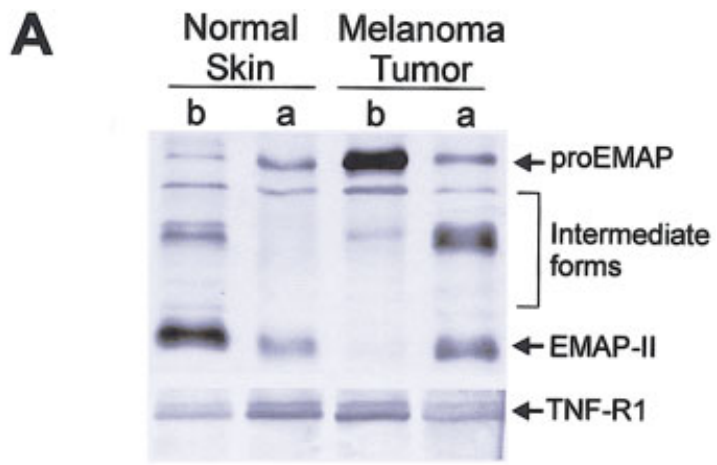

B
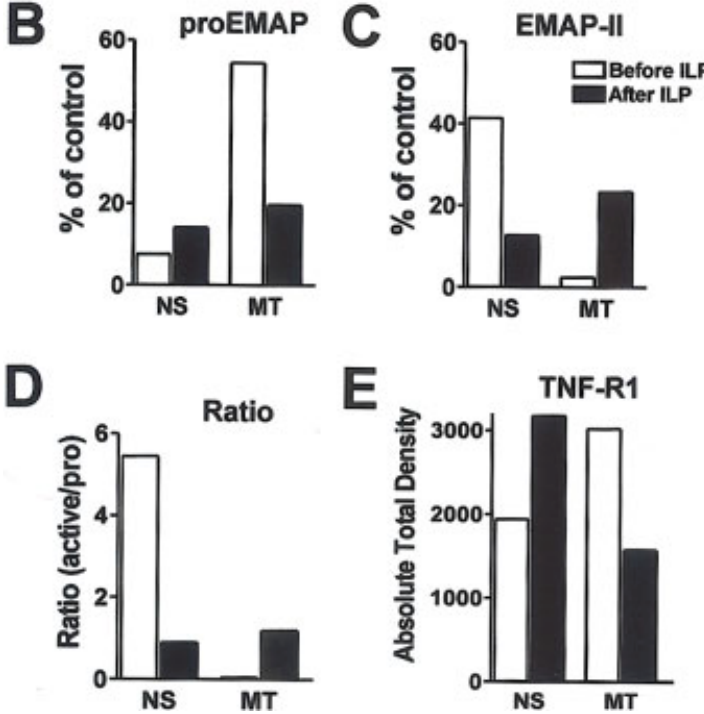

FIgURE 6 - Comparison of EMAP-II and TNF-R1 expression in tumour biopsies and normal skin tissue from a melanoma patient before (b) and after (a) TNF-based ILP. (a) Western blots. (b) Quantification of proEMAP expression before (open bars) and after (closed bars) ILP as compared to the positive control. (c) Quantification of EMAP-II expression before and after ILP as compared to the positive control. (d) Ratio of EMAP-II/proEMAP derived from $(b)$ and $(c)$ before and after ILP. (e) Quantification of TNF-R1 expression before and after ILP shown as absolute density values. For EMAP-II and TNF-R1 in the normal skin exactly the opposite is occurring as compared to the melanoma tumour. Abbreviations: NS, normal skin; MT, melanoma tumour.

ence between endothelial cells in culture and those of tumour vessels. The fact that there is no co-staining of endothelial cells and TNF-R1 shows that the underlying mechanism responsible for the TNF response is much more multifaceted than the simple binding of TNF to TNF-R1 on endothelial cells resulting in endothelial cell apoptosis. We speculate that during TNF-based ILP a whole set of cytokines, growth factors and cells are involved, thereby changing the tumour pathophysiology, like cellular organization and vascular flow, which opens windows for TNF and melphalan to exert their anti-tumour activities.

Experiments with co-staining for macrophages confirm this hypothesis and pinpoint macrophages and EMAP-II as important players during TNF-based ILP. The tumour-associated macrophages are located in regions of high EMAP-II expression and are attracted by the chemo attractive properties of EMAP-II to vascular structures before ILP (Fig. 5b). Also after ILP there is still a strong co-localization of EMAP-II and macrophages, which implies that EMAP-II is still after ILP directing macrophages to specific sites. These sites are regions of vascular remnants as revealed by co-staining of endothelial cells and macrophages (Fig. 5c). While before ILP the vessels are intact and the macrophages are spread throughout the tumour, after ILP the macrophages are recruited to sites of vascular destruction. Our data show that EMAP-II is an important regulator of this process. For TNF-R1 we observed co-staining with macrophages only before ILP. This might indicate a role for TNF-R1 in activating the tumour-associated macrophages during their suggested role in tumour response.

These observations, the fact that TNF targets endothelial cells within solid tumours and that all these observations were made directly after ILP imply that TNF attributes mainly to these phenomena. Unfortunately, no control tissue was available of (melanoma) patients treated with Melphalan alone for verification, because all patients get the optimal TNF + Melphalan treatment. Additionally, the anti-vascular and cytokine dependent (macrophage) effects will be much lower in the melphalan alone group making adequate evaluation complicated.

The opposing effect of ILP treatment on proEMAP-EMAP-II in the normal skin as compared to the melanoma tumour (Fig. 6) is a remarkable finding but only done in 1 patient. TNF selectively disrupts tumour vasculature; maybe the effects on EMAP-II and macrophages recruitment may be tumour specific. The high EMAP-II expression in the normal skin can be explained by the high level of cell turnover and replacement, which occurs in healthy skin tissue. ${ }^{31}$

Besides the observed EMAP-II effect on macrophages during ILP treatment, an important observation of our study is the relationship between EMAP-II expression and CR of melanoma patients after TNF-based ILP. Particularly, the correlation was found for the active cytokine EMAP-II, and not for proEMAP, ratio or TNF-R1 (Table II). This correlation between EMAP-II and clinical response strongly suggests an important function of EMAP-II both in mechanism and in clinical outcome. For sarcomas no correlation was found. The relatively small group of patients we studied $(n=14)$ may be responsible for this but moreover, sarcomas are very heterogeneous tumours and our group consists of many different subtypes. In addition, sarcoma tumours had a lower EMAP-II expression as compared to melanomas and depending on tumour type EMAP-II is expressed at different levels and by different mechanisms. ${ }^{12}$ A role of EMAP-II in sarcoma regression is not ruled out but for sarcomas additional factors might play crucial roles. We speculate that a certain threshold of EMAP-II needs to be reached to establish its reported effects and this likely is strongly dependent on tumour type and tumour micro-environmental composition like matrix composition, infiltration and vascular density. Our results justify further experimental analysis of the function of EMAP-II in anti-tumour activities of TNF. This will lead to more insight in this process and possibilities to intervene and manipulate the EMAP-II expression or activity improving response rates in the clinical setting. In conclusion, we propose that analysis of EMAP-II expression in tumour biopsies of melanoma patients should be implemented in the ILP-procedure so this correlation could be defined in a randomised trial within a larger patient group.

\section{References}

1. Eggermont AM, de Wilt JH, ten Hagen TL. Current uses of isolated limb perfusion in the clinic and a model system for new strategies. Lancet Oncol 2003;4:429-37.

2. Eggermont AM, Schraffordt KH, Lienard D, Kroon BB, van Geel AN, Hoekstra HJ, Lejeune FJ. Isolated limb perfusion with high-dose tumor necrosis factor- $\alpha$ in combination with interferon- $\beta$ and melpha- lan for nonresectable extremity soft tissue sarcomas: a multicenter trial. J Clin Oncol 1996;14:2653-65.

3. Eggermont AM, Schraffordt KH, Klausner JM, Kroon BB, Schlag PM, Lienard D, van Geel AN, Hoekstra HJ, Meller I, Nieweg OE, Kettelhack C, Ben Ari G et al. Isolated limb perfusion with tumor necrosis factor and melphalan for limb salvage in 186 patients with 
locally advanced soft tissue extremity sarcomas. The cumulative multicenter European experience. Ann Surg 1996;224:756-64.

4. Lejeune FJ, Lienard D, Eggermont AMM, Schraffordt Koops H, Rosenkaimer F, Gerain J, Klaase JM, Kroon BBR, Vanderveken J, Schmitz P. Rationale for using TNF $\alpha$ and chemotherapy in regional therapy of melanoma. J Cell Biochem 1994;56:52-61.

5. Fraker DL, Alexander HR, Andrich M, Rosenberg SA. Treatment of patients with melanoma of the extremity using hyperthermic isolated limb perfusion with melphalan, tumor necrosis factor, and interferon $\beta$ : results of a tumor necrosis factor dose-escalation study. J Clin Oncol 1996;14:479-89.

6. Kao J, Ryan J, Brett G, Chen J, Shen H, Fan YG, Godman G, Familletti PC, Wang F, Pan YC, et al. Endothelial monocyte-activating polypeptide II. A novel tumor-derived polypeptide that activates hostresponse mechanisms. J Biol Chem 1992;267:20239-47.

7. Murray JC, Clauss M, Denekamp J, Stern D. Selective induction of endothelial cell tissue factor in the presence of a tumour-derived mediator: a potential mechanism of flavone acetic acid action in tumour vasculature. Int J Cancer 1991;49:254-9.

8. Clauss M, Murray JC, Vianna M, de Waal R, Thurston G, Nawroth P, Gerlach H, Bach R, Familletti PC, Stern D. A polypeptide factor produced by fibrosarcoma cells that induces endothelial tissue factor and enhances the procoagulant response to tumor necrosis factor/cachectin. J Biol Chem 1990;265:7078-83.

9. Kao J, Fan YG, Haehnel I, Brett J, Greenberg S, Clauss M, Kayton M, Houck K, Kisiel W, Seljelid R, et al. A peptide derived from the amino terminus of endothelial-monocyte-activating polypeptide II modulates mononuclear and polymorphonuclear leukocyte functions, defines an apparently novel cellular interaction site, and induces an acute inflammatory response. J Biol Chem 1994:269:9774-82.

10. Kao J, Houck K, Fan Y, Haehnel I, Libutti SK, Kayton ML, Grikscheit T, Chabot J, Nowygrod R, Greenberg S et al. Characterization of a novel tumor-derived cytokine. Endothelial-monocyte activating polypeptide II. J Biol Chem 1994:269:25106-19.

11. Schwarz MA, Kandel J, Brett J, Li J, Hayward J, Schwarz RE, Chappey O, Wautier JL, Chabot J, Lo Gerfo P, Stern D. Endothelial-monocyte activating polypeptide II, a novel antitumor cytokine that suppresses primary and metastatic tumor growth and induces apoptosis in growing endothelial cells. J Exp Med 1999;190:341-54.

12. Murray JC, Symonds P, Ward W, Huggins M, Tiga A, Rice K, Heng YM, Todd I, Robins RA. Colorectal cancer cells induce lymphocyte apoptosis by an endothelial monocyte-activating polypeptide-II-dependent mechanism. J Immunol 2004;172:274-81.

13. Shalak V, Kaminska M, Mitnacht-Kraus R, Vandenabeele P, Clauss M, Mirande M. The EMAPII cytokine is released from the mammalian multisynthetase complex after cleavage of its p43/proEMAPII component. J Biol Chem 2001;276:23769-76.

14. Gnant MF, Berger AC, Huang J, Puhlmann M, Wu PC, Merino MJ, Bartlett DL, Alexander HR, Jr, Libutti SK. Sensitization of tumor necrosis factor $\alpha$-resistant human melanoma by tumor-specific in vivo transfer of the gene encoding endothelial monocyte-activating polypeptide II using recombinant vaccinia virus. Cancer Res 1999;59: 4668-74.

15. Wu PC, Alexander HR, Huang J, Hwu P, Gnant M, Berger AC, Turner E, Wilson O, Libutti SK. In vivo sensitivity of human melanoma to tumor necrosis factor (TNF)- $\alpha$ is determined by tumor production of the novel cytokine endothelial-monocyte activating polypeptide II (EMAPII). Cancer Res 1999;59:205-12.
16. Berger AC, Alexander HR, Wu PC, Tang G, Gnant MF, Mixon A, Turner ES, Libutti SK. Tumour necrosis factor receptor I (p55) is upregulated on endothelial cells by exposure to the tumour-derived cytokine endothelial monocyte- activating polypeptide II (EMAP-II). Cytokine 2000;12:992-1000.

17. Tandle AT, Mazzanti C, Alexander HR, Roberts DD, Libutti SK. Endothelial monocyte activating polypeptide-II induced gene expression changes in endothelial cells. Cytokine 2005;30:347-58

18. Lans TE, ten Hagen TL, van Horssen R, Wu PC, van Tiel ST, Libutti SK, Alexander HR, Eggermont AM. Improved antitumor response to isolated limb perfusion with tumor necrosis factor after upregulation of endothelial monocyte-activating polypeptide II in soft tissue sarcoma. Ann Surg Oncol 2002;9:812-9.

19. WHO. WHO handbook for reporting results of cancer treatment. Geneva: World Health Organisation, 1979.

20. Barnett G, Jakobsen AM, Tas M, Rice K, Carmichael J, Murray JC Prostate adenocarcinoma cells release the novel proinflammatory polypeptide EMAP-II in response to stress. Cancer Res 2000;60:2850-7.

21. Murray JC, Barnett G, Tas M, Jakobsen A, Brown J, Powe D, Clelland C. Immunohistochemical analysis of endothelial-monocyteactivating polypeptide-II expression in vivo. Am J Pathol 2000;157: 2045-53.

22. Grunhagen DJ, Brunstein F, Graveland WJ, van Geel AN, de Wilt JH, Eggermont AM. One hundred consecutive isolated limb perfusions with TNF- $\alpha$ and melphalan in melanoma patients with multiple intransit metastases. Ann Surg 2004;240:939-47.

23. Clarijs R, Schalkwijk L, Ruiter DJ, de Waal RM. EMAP-II expression is associated with macrophage accumulation in primary uveal melanoma. Invest Ophthalmol Vis Sci 2003;44:1801-6.

24. Aderka D, Sorkine P, Abu-Abid S, Lev D, Setton A, Cope AP, Wallach D, Klausner J. Shedding kinetics of soluble tumor necrosis factor (TNF) receptors after systemic TNF leaking during isolated limb perfusion. Relevance to the pathophysiology of septic shock. J Clin Invest 1998;101:650-9.

25. Gerain J, Lienard D, Pampallona S, Baumgartner M, Ruegg C, Buurman WA, Eggermont A, Lejeune F. Systemic release of soluble TNF receptors after high-dose TNF in isolated limb perfusion. Cytokine 1997:9:1034-42.

26. Siegel RM, Muppidi JR, Sarker M, Lobito A, Jen M, Martin D, Straus SE, Lenardo MJ. SPOTS: signaling protein oligomeric transduction structures are early mediators of death receptor-induced apoptosis at the plasma membrane. J Cell Biol 2004;167:735-44.

27. Berger AC, Tang G, Alexander HR, Libutti SK. Endothelial monocyte-activating polypeptide II, a tumor-derived cytokine that plays an important role in inflammation, apoptosis, and angiogenesis. J Immunother 2000;23:519-27.

28. Bingle L, Brown NJ, Lewis CE. The role of tumour-associated macrophages in tumour progression: implications for new anticancer therapies. J Pathol 2002;196:254-65.

29. Tas MP, Murray JC. Endothelial-monocyte-activating polypeptide II Int J Biochem Cell Biol 1996;28:837-41.

30. Jones SJ, Ledgerwood EC, Prins JB, Galbraith J, Johnson DR, Pober JS, Bradley JR. TNF recruits TRADD to the plasma membrane but not the trans-Golgi network, the principal subcellular location of TNF-R1. J Immunol 1999:162:1042-8.

31. Park SG, Shin H, Shin YK, Lee Y, Choi EC, Park BJ, Kim S. The novel cytokine p43 stimulates dermal fibroblast proliferation and wound repair. Am J Pathol 2005;166:387-98. 